\title{
4-Chlorophenyl and 2,4,6-tribromophenyl chlorothionoformates: new dealkylating agents for tertiary amines
}

\author{
Mahmoud Heidari and Mehdi M. Baradarani* \\ Department of Chemistry, Faculty of Science, University of Urmia, Urmia 57135, Iran \\ E-mail: m.baradarani@mail.urmia.ac.ir
}

\begin{abstract}
4-Chlorophenyl and 2,4,6-tribromophenyl chlorothionoformates react rapidly with tertiary amines at $20^{\circ} \mathrm{C}$ to give a thiocarbamate and alkyl chloride. The thiocarbamates are converted to the secondary amine salts by treatment with dimethyl sulfate, followed by hydrolysis with water. Rates of reaction, and alkyl group clevage selectivity in amines were found to be comparable or superior to those previously reported with chloroformates.
\end{abstract}

Keywords: 4-Chlorophenyl chlorothionoformate, 2,4,6-tribromophenyl chloro thiono- formate

\section{Introduction}

We have previously reported the epimerization of phthalideisoquinoline alkaloid $^{1}$ with thiophosgene and the dealkylation of tertiary aliphatic amines with thiophosgene and 1chloroethyl chlorothionoformate ${ }^{2}$. The reactivity of these reagents were found to be comparable to that of a number of previously used methods, such as the Von Braun ${ }^{3}$ reaction or the use of ethyl chloroformate ${ }^{4}$ and phenyl chloroformate ${ }^{5}$. The latter reagents produce products which are difficult to hydrolysis, and accordingly were replaced by 1-chloroethyl chloroformate ${ }^{6}, 2,2,2$ trichloroethyl chloroformate ${ }^{7}$ and vinyl chloroformate . $^{8}$.

We have found that 4-chlorophenyl chlorothionoformate and 2,4,6-tribromophenyl chlorothionoformate are even more reactive towards amines than chloroformate and thiophosgene, but the ease and selectivity of the analogous second dealkylation step required elaboration. ( Scheme 1 ) 


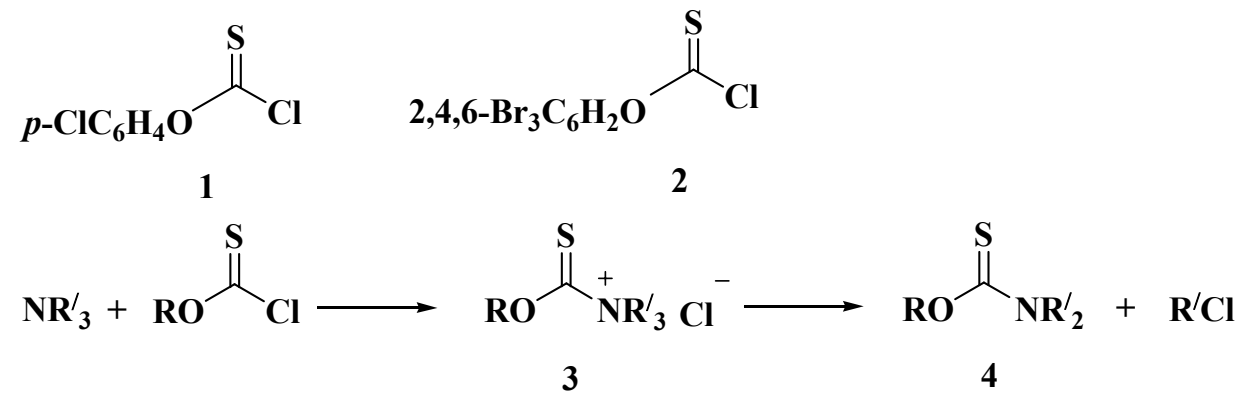

\section{Scheme 1}

In this paper we aim to ascertain the relative rates of cleavage of alkyl groups with 4chlorophenyl chlorothionoformate and 2,4,6-tribromophenyl chlorothionoformate to determine the scope of their effectivness as a dealkylating agents for tertiary aliphatic amines.

\section{Result and Discussion}

The accepted pathway ${ }^{9}$ for dealkylation of tertiary amines with chloroformates involves the rate determining decomposition of the salt (3) by chloride, forming the carbamate (4) and an alkyl chloride. In a general way this appears also to be the case with chlorothiono-formates. The reaction of unhindered tertiary amine with 4-chlorophenyl chlorothionoformates was very rapid at $20^{\circ} \mathrm{C}$ in dichloromethane as a solvent. When the reaction of triethylamine with an equivalent of (1) was followed by ${ }^{1} \mathrm{H}$ n.m.r analysis, the ammonium salt appeared to form instantly $\left(\delta \mathrm{CH}_{2}\right.$ $4.35 \mathrm{ppm}$ ), and chloroethane formation could be followed to completion over the next $40 \mathrm{~min}$. As shown in table $\mathbf{1}$ and table $\mathbf{2}$, the reaction of various tertiary amines were complete within 40 minutes by the use of 4-chlorophenyl chlorothionoformate (1) and two hours when 2,4,6tribromophenyl chlorothionoformate was used.

Table 1. Dealkylation of tertiary amines with (1)

\begin{tabular}{lccc}
\hline Amine & \multicolumn{1}{c}{ Condition } & Product & \%Yield \\
\hline 1. Triethylamine & $\mathrm{CH}_{2} \mathrm{Cl}_{2}, 40 \mathrm{~min}, 20{ }^{\circ} \mathrm{C}$ & $\mathbf{5 a}$ & 95 \\
2. N,N-Dimethyl benzyl & $\mathrm{CH}_{2} \mathrm{Cl}_{2}, 40 \mathrm{~min}, 20^{\circ} \mathrm{C}$ & $\mathbf{6 a}$ & 97 \\
amine & & & \\
3.1-Methylpiperidine & $\mathrm{CH}_{2} \mathrm{Cl}_{2}, 40 \mathrm{~min}, 20{ }^{\circ} \mathrm{C}$ & $\mathbf{8 a}$ & 96 \\
4. 4-Methylmorpholine & $\mathrm{CH}_{2} \mathrm{Cl}_{2}, 40 \mathrm{~min}, 20^{\circ} \mathrm{C}$ & $\mathbf{9 a}$ & 96 \\
5.1-Methylpyrrolidine & $\mathrm{CH}_{2} \mathrm{Cl}_{2}, 40 \mathrm{~min}, 20^{\circ} \mathrm{C}$ & $\mathbf{1 0 a}$ & 96 \\
6.(-)-Nicotine & $\mathrm{CH}_{2} \mathrm{Cl}_{2}, 40 \mathrm{~min}, 20^{\circ} \mathrm{C}$ & $\mathbf{1 1 a}$ & 95 \\
7. N,N-Dimethylaniline & neat, $24 \mathrm{~h}, 130^{\circ} \mathrm{C}$ & $\mathbf{7 a}$ & 75 \\
\hline
\end{tabular}


Table 2. Dealkylation of tertiary amines with (2)

\begin{tabular}{lccc}
\hline Amine & Condition & Product & \%Yield \\
\hline 1. Triethylamine & $\mathrm{CH}_{2} \mathrm{Cl}_{2}, 2 \mathrm{~h}, 20{ }^{\circ} \mathrm{C}$ & $\mathbf{5 b}$ & 91 \\
2. N,N-Dimethyl benzyl & $\mathrm{CH}_{2} \mathrm{Cl}_{2}, 2 \mathrm{~h}, 20{ }^{\circ} \mathrm{C}$ & $\mathbf{6 b}$ & 96 \\
amine & & & \\
3.1-Methylpiperidine & $\mathrm{CH}_{2} \mathrm{Cl}_{2}, 2 \mathrm{~h}, 20{ }^{\circ} \mathrm{C}$ & $\mathbf{8 b}$ & 93 \\
4. 4-Methylmorpholine & $\mathrm{CH}_{2} \mathrm{Cl}_{2}, 2 \mathrm{~h}, 20^{\circ} \mathrm{C}$ & $\mathbf{9 b}$ & 83 \\
5.1-Methylpyrrolidine & $\mathrm{CH}_{2} \mathrm{Cl}_{2}, 2 \mathrm{~h}, 20^{\circ} \mathrm{C}$ & $\mathbf{1 0 b}$ & 89 \\
6. N,N-Dimethylaniline & neat, $24 \mathrm{~h}, 130^{\circ} \mathrm{C}$ & $\mathbf{7 b}$ & 71 \\
\hline
\end{tabular}
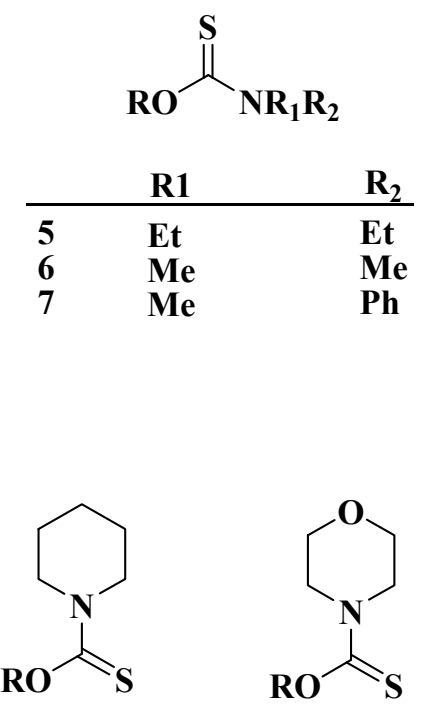

8<smiles>[R20]C(=S)N1CCOCC1</smiles>

9<smiles>[R20]C(=S)N(C)CCCCCl</smiles>

10

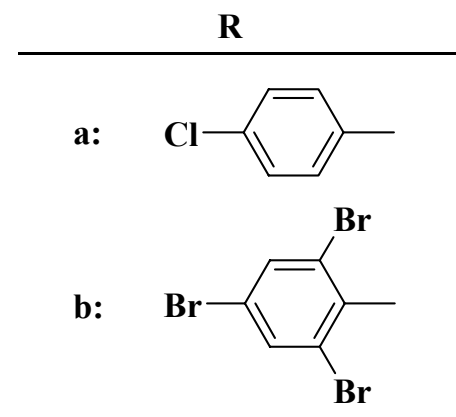<smiles>[R]OC(=S)N(C)CCCC(Cl)c1cccnc1</smiles>

11

Olofson $^{8}$ has used $N$-methylpiperidine as the benchmark for the determination of rate and selectivity of the dealkylation of tertiary amines by vinyl chloroformate, cyanogen bromide, phenylchloroformate, ethyl chloroformate, 2,2,2-trichloroethyl chloroformate and benzyl chloroformate. The use of vinyl chloroformate give the demethylated product in $90 \%$ yield but a period at $80^{\circ} \mathrm{C}$ was required to effect decomposition of the intermediate salt.

The use of 4-chlorophenyl chlorothionoformate and even a hindered derivative like 2,4,6tribromophenyl chlorothionoformate resulted in dealkylation at $20^{\circ} \mathrm{C}$, while the other chlorofromates effected the conversion in, at best, $10 \%$ yield, under similar conditions. Cyanogen bromide showed low selectivity, giving cyanamide (12) (54\%), accompanied by the ring opened (13) (28\%). We reported ${ }^{2}$ that the reaction of 1-chloroethyl chlorothionoformate with $N$-methylpiperidine afforded the carbamate (14) in $75 \%$ yield at reflux for 12 hours in dichloromethane. Similarly, the reaction of 1-chloroethyl-chloroformate with $N$-methylpiperidine gave the carbamate $(\mathbf{1 5})$ at $80^{\circ} \mathrm{C}$. 


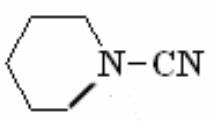

12

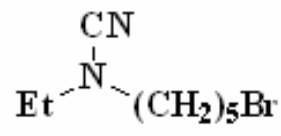

13

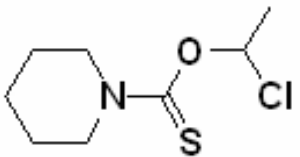

14

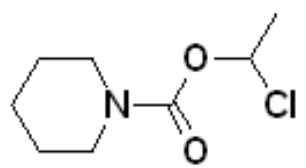

15

By contrast, 4-chlorophenyl and even 2,4,6-tribromophenyl chlorothionoformates cleanly converted triethylamine and $N$-methylpiperidine to the corresponding thiocarbamate $(\mathbf{8})$ at room temperature within 40 min (1) or two hours (2). Thus demonstrating agent the reactivity of $\mathbf{1}$ and 2 with tertiary amines is superior to that of vinylchloroformate, 1-chloroethyl chloroformate and the 1-chloroethyl chlorothiono-formate. Selectivity of the cleavage of alkyl group by chlorothionoformates were also investigated. Reaction of $N, N$-dimethylbenzylamine with (1) and (2) resulted only in debenzylation, at room temperature in almost quantitative yield. While Nmethyl-morpholine undergoes considerable ring opening on reaction with either cyanogen bromide ${ }^{10}$ or chlorothionoformate, only the demethylation was observed. Demethylation of $N, N-$ dimethyl-aniline with (1) and (2)were very slow, but the demethylated product (7) were isolated in $75 \%$ and $71 \%$ yields after reaction at $130^{\circ} \mathrm{C}$ for $24 \mathrm{~h}$; a similar reaction, with phenyl chloroformate $^{5}$ gave $80 \%$ yield at $100^{\circ} \mathrm{C}$ after $60 \mathrm{~h}$. When $\mathrm{N}$-methylpyrrolidine as a five membered ring amine was treated with (1) in dichloromethan the reaction proceeded to completion rapidly at $20^{\circ} \mathrm{C}$, but ring opening was achieved leading to (10a) in $96 \%$ yield, as two rotamers by ${ }^{1} \mathrm{H}$ and ${ }^{13} \mathrm{C} \mathrm{nmr}$ spectroscopy. Similarly ring openning product (10b) as two rotamers was produced in $89 \%$ yield from the reaction of $N$-methylpyrrolidine with (2) at $20^{\circ} \mathrm{C}$ for two hours. When (-)-nicotine was treated with (1) and (2) at $20^{\circ} \mathrm{C}$, the ring opened products 11a and 11b were formed in 89 and $86 \%$ yields, respectively.

In conclusion 4-chlorophenyl chlorothionoformate (1) and 2,4,6-tribromophenyl chlorothionoformate (2) are shown to be useful dealkylating agents for tertiary amine. The dialkylthiocarbamates could be readily converted to the corresponding amines by sequential treatment with dimethylsulfate followed by their hydrolysis in water. (Scheme 2)

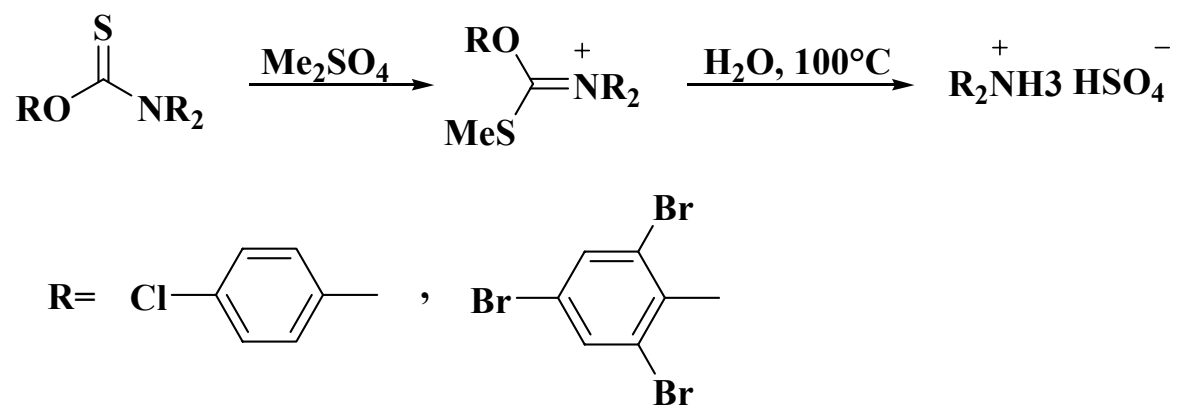

\section{Scheme 2}




\section{Experimental Section}

General Procedures. ${ }^{1} \mathrm{H}$ and ${ }^{13} \mathrm{C}$ n.m.r spectra were recorded with a Bruker spectrometer in $\mathrm{CDCl}_{3}$ at $300 \mathrm{MHz}$ unless otherwise indicated and chemical shifts are in ppm. Infrared spectra were recorded on a Thermonicolet-Nexus 670 FT-IR spectrometer, with sampels measured as a neat film (oil) or $\mathrm{KBr}$ disk (solid). Mass spectra were recorded on a Agilent 6890-N-NetworkGC-system. Melting points were determined on a Philip Harris C4954718 apparatus and remain uncorrected.

Analytical thin-layer chromatography (TLC) was carried out with Merck silica gel 60 $\mathrm{F}_{254 \lambda}$ aluminium sheets. The identity of previously prepared compounds was confirmed by comparison of spectra data with that of published. In all casses isolation of only a single thiocarbamate is reported, n.m.r analysis of the crude product mixture confirmed that no other thiocarbamate was produced.

The routine purification of reagents and solutions was carried out by standard laboratory procedures. All organic extracts were dried with anhydrous sodium sulfate.

Typical reaction of tertiary amine with 1. 4-Chlorophenyl chlorothionoformate ${ }^{12}$ (1) (1.02g, $4.94 \mathrm{mmol})$ was added to a solution of tertiary amine $(4.94 \mathrm{mmol})$ in dichloromethane $(20 \mathrm{ml})$ and the solution stirred under nitrogen at $20^{\circ} \mathrm{C}$ for 40 minutes. The solvent was evaporated affording a crude oily product which was purified by chromatography (pet. ether:dichloromethan 20:1) on silica.

$\boldsymbol{O}$-4-Chlorophenyl diethylthiocarbamate (5a). White crystals, 95\% yield, m.p. $76-79{ }^{\circ} \mathrm{C} .{ }^{1} \mathrm{H}$ NMR $\delta 1.33\left(\mathrm{t}, J=7.2,6 \mathrm{H}, 2 \mathrm{CH}_{3}\right), 3.69$ (q, $\left.J=7.2,2 \mathrm{H}, \mathrm{CH}_{2}\right), 3.90\left(\mathrm{q}, J=7.2,2 \mathrm{H}, \mathrm{CH}_{2}\right), 7.02(\mathrm{~d}$, $J=9,2 \mathrm{H}, \mathrm{Ar}), 7.36(\mathrm{~d}, J=9,2 \mathrm{H}, \mathrm{Ar}) ;{ }^{13} \mathrm{C} \mathrm{NMR} \delta 11.79,13.55,44.33,48.49,124.22,129.22$, 131.24, 152.38, 186.48; FT-IR $v_{\max }$ : 2975, 1514, 1484, 1428, 1312, 1284, 1213, 1160, 1128 , 1081, 832, $692 \mathrm{~cm}^{-1}$; MS : m/z : 242(M $\left.\mathrm{M}^{+}\right), 116,100(100), 88,72,60$.

$\boldsymbol{O}$-4-Chlorophenyl dimethylthiocarbamate (6a). White crystals, 97\% yield, m.p. $\quad$ 50$51^{\circ} \mathrm{C} .{ }^{1} \mathrm{H}$ NMR $\delta 3.29\left(\mathrm{~s}, 3 \mathrm{H}, \mathrm{CH}_{3}\right), 3.40\left(\mathrm{~s}, 3 \mathrm{H}, \mathrm{CH}_{3}\right), 7.00(\mathrm{~d}, J=8.7,2 \mathrm{H}, \mathrm{Ar}), 7.33(\mathrm{~d}, J=8.7$, $2 \mathrm{H}, \mathrm{Ar}) ;{ }^{13} \mathrm{C}$ NMR $\delta 38.84,43.34,124.31,129.20,131.15,152.53,187.25 ; \quad$ FT-IR $v_{\max }: 2934$, $1536,1483,1395,1287,1211,1140,1081,1010,836,700 \mathrm{~cm}^{-1} ; \mathrm{MS}: \mathrm{m} / \mathrm{z}: 215\left\{\mathrm{M}^{+}\right), 88$, $72(100), 44$.

$\boldsymbol{O}$-4-Chlorophenyl piperidine-1-carbothioate (8a). Milky crystals, 96\% yield, m.p. $\quad$ 54$55^{\circ} \mathrm{C} .{ }^{1} \mathrm{H}$ NMR $\delta$ 1.72(bs, $\left.6 \mathrm{H}, 3 \mathrm{CH}_{2}\right), 3.90\left(\mathrm{bs}, 2 \mathrm{H}, \mathrm{CH}_{2}\right), 4.10\left(\mathrm{bs}, 2 \mathrm{H}, \mathrm{CH}_{2}\right), 7.00(\mathrm{~d}, J=7.8,2 \mathrm{H}$, Ar), 7.34(d, $J=7.8,2 \mathrm{H}, \mathrm{Ar}) ;{ }^{13} \mathrm{C}$ NMR $\delta 24.16,25.26,26.09,47.56,51.84,124.26,129.17$, 131.16, 152.47, 185.76; FT-IR $v_{\max }$ : 2937, 2853, 1499, 1443, 1283, 1206, 1165, 1146, 1011, 836, $694 \mathrm{~cm}^{-1} ; \mathrm{MS}: \mathrm{m} / \mathrm{z}: 255\left(\mathrm{M}^{+}\right), 128,112(100), 69,41$.

O-4-chlorophenyl morpholine-4-carbothioate (9a). White crystals , 96\% yield, m.p. 96-98 ${ }^{\circ}$. ${ }^{1} \mathrm{H}$ NMR $\delta 3.78\left(\mathrm{t}, J=4.8,2 \mathrm{H}, \mathrm{CH}_{2}-\mathrm{N}\right), 3.84\left(\mathrm{t}, J=4.5,2 \mathrm{H}, \mathrm{CH}_{2}-\mathrm{N}\right), 3.97\left(\mathrm{t}, J=4.8,2 \mathrm{H}, \mathrm{CH}_{2}-\mathrm{O}\right)$, 4.15(t, $\left.J=4.8,2 \mathrm{H}, \mathrm{CH}_{2}-\mathrm{O}\right), 7.14(\mathrm{~d}, J=8.7,2 \mathrm{H}, \mathrm{Ar}), 7.37(\mathrm{~d}, J=8.7,2 \mathrm{H}, \mathrm{Ar}) ;{ }^{13} \mathrm{C}$ NMR $\delta$ 46.82, 50.24, 66.09, 66.32, 124.16, 129.33, 131.54, 152.18, 186.85; FT-IR $v_{\max }$ : 2869, 1487, 1443, 1283, 1244, 1205, 1114, 1082, 1037, $836 \mathrm{~cm}^{-1}$; MS : m/z : 257(M $\left.\mathrm{M}^{+}\right), 130,114(100), 86,70$. 
$\boldsymbol{O}$-4-Chlorophenyl 4-chlorobutyl(methyl)carbamothioate (10a). Yellow oil , 96\% yield, ${ }^{1} \mathrm{H}$ NMR $\delta$ (rotamer 1) $1.83\left(\mathrm{~m}, 4 \mathrm{H}, 2 \mathrm{CH}_{2}\right), 3.26\left(\mathrm{~s}, 3 \mathrm{H}, \mathrm{CH}_{3}\right), 3.54-3.62\left(\mathrm{~m}, 2 \mathrm{H}, \mathrm{CH}_{2}-\mathrm{N}\right), 3.72(\mathrm{t}, J=$ $\left.7.2,2 \mathrm{H}, \mathrm{CH}_{2}-\mathrm{Cl}\right), 6.99(\mathrm{~d}, J=7.2,2 \mathrm{H}, \mathrm{Ar}), 7.33(\mathrm{~d}, J=6.9,2 \mathrm{H}, \mathrm{Ar}) ;{ }^{1} \mathrm{H} \mathrm{NMR} \delta$ (rotamer 2) 1.83(m, $\left.4 \mathrm{H}, 2 \mathrm{CH}_{2}\right), 3.38\left(\mathrm{~s}, 3 \mathrm{H}, \mathrm{CH}_{3}\right), 3.54-3.62\left(\mathrm{~m}, 2 \mathrm{H}, \mathrm{CH}_{2}-\mathrm{N}\right), 3.89\left(\mathrm{t}, J=7.2,2 \mathrm{H}, \mathrm{CH}_{2}-\mathrm{Cl}\right)$, $6.99(\mathrm{~d}, J=7.2,2 \mathrm{H}, \mathrm{Ar}), 7.33(\mathrm{~d}, J=6.9,2 \mathrm{H}, \mathrm{Ar})$;

${ }^{13} \mathrm{C}$ NMR $\delta$ (rotamer 1) 23.81, 29.59, 36.85, 44.42, 50.81, 124.24, 129.17, 131.17, 152.42, 187.16. ${ }^{13} \mathrm{C}$ NMR $\delta$ (rotamer 2) 25.27, 29.59, 41.65, 44.70, 54.53, 124.39, 129.26, 131.22, 152.42, 187.31; FT-IR $v_{\max }: 2943,1517,1485,1401,1208,1132,1085,1014,833 \mathrm{~cm}^{-1}$; MS : $\mathrm{m} / \mathrm{z}: 291\left(\mathrm{M}^{+}\right), 148,91(100), 74,55$.

O-4-Chlorophenyl 4-chloro-4-(pyridin-3-yl)butyl(methyl)carbamothioate (11a). Yellowish orange oil , 95\% yield, ${ }^{1} \mathrm{H}$ NMR (rotamer 1) $\left(\mathrm{CDCl}_{3}\right) \delta(\mathrm{ppm}) 1.70-2.20\left(\mathrm{~m}, 4 \mathrm{H}, 2 \mathrm{CH}_{2}\right), 3.22(\mathrm{~s}$, $\left.3 \mathrm{H}, \mathrm{CH}_{3}\right), 3.70\left(\mathrm{t}, J=7.2,2 \mathrm{H}, \mathrm{CH}_{2}-\mathrm{N}\right), 4.88-4.98(\mathrm{~m}, 1 \mathrm{H}, \mathrm{CH}-\mathrm{Cl}), 6.89-6.98(\mathrm{~m}, 2 \mathrm{H}, \mathrm{Ar}), 7.20-$ 7.33(m, 3H, Ar), 7.67-7.75(m, 1H, Ar), 8.53-8.61(m, 2H, Ar); ${ }^{1} \mathrm{H}$ NMR (rotamer 2) $\left(\mathrm{CDCl}_{3}\right) \delta$ (ppm) 1.70-2.20(m, 4H, 2CH$), 3.34\left(\mathrm{~s}, 3 \mathrm{H}, \mathrm{CH}_{3}\right), 3.78-4.09\left(\mathrm{~m}, 2 \mathrm{H}, \mathrm{CH}_{2}-\mathrm{N}\right), 4.88-4.98(\mathrm{~m}, 1 \mathrm{H}$, $\mathrm{CH}-\mathrm{Cl}), 6.89-6.98(\mathrm{~m}, 2 \mathrm{H}, \mathrm{Ar}), 7.20-7.33(\mathrm{~m}, 3 \mathrm{H}, \mathrm{Ar}), 7.67-7.75(\mathrm{~m}, 1 \mathrm{H}, \mathrm{Ar}), 8.53-8.61(\mathrm{~m}, 2 \mathrm{H}$, $\mathrm{Ar}) ;{ }^{13} \mathrm{C} \mathrm{NMR}$ (rotamer1) $\left(\mathrm{CDCl}_{3}\right) \delta(\mathrm{ppm}) 23.88,36.62,36.78,50.69,59.96,123.75,124.13$, $129.20,131.27,134.43,136.85,148.24,149.70,152.27,187.20 ;{ }^{13} \mathrm{C} \mathrm{NMR}$ (rotamer 2$)\left(\mathrm{CDCl}_{3}\right) \delta$ (ppm) 25.31, 36.74, 41.69, 54.26, 60.21, 123.75, 124.25, 129.29, 131.34, 134.55, 137.14, 148.34, 149.87, 152.30, 187.46; FT-IR $v_{\max }$ (KBr disk): 2938, 1518, 1485, 1402, 1208, 1136, 1085, 834, $713 \mathrm{~cm}^{-1}$; MS : m/z : 369(M+), 225(100), 197, 168.

Reaction of $\mathbf{N}, \mathbf{N}$-dimethylaniline with (1). The mixture of $\mathrm{N}, \mathrm{N}$-dimethylaniline $(0.5 \mathrm{~g}, 0.52 \mathrm{ml}$, $4.12 \mathrm{mmol})$ and 4-chlorophenyl chlorothionoformate (1) $(0.854 \mathrm{~g}, 4.12 \mathrm{mmol})$ were heated under nitrogen at $130^{\circ} \mathrm{C}$ for 24 hours to give a blue oil. The resulted oily product was purified by chromatography (pet. ether:ethyl acetate 20:1) and O-4-chloro-phenyl methyl(phenyl)thiocarbamate (7a) was separated as a yellow crystals $(0.86 \mathrm{~g}, 75 \%)$, m.p.107$110^{\circ} \mathrm{C}$.

${ }^{1} \mathrm{H}$ NMR $\delta$ 3.71(s, 3H, CH$), 7.04-7.77(\mathrm{~m}, 9 \mathrm{H}, \mathrm{Ar}-\mathrm{H}) ;{ }^{13} \mathrm{C}$ NMR $\delta 44.94,124.12,125.64$, 127.94, 129.29, 129.60, 131.30, 143.41, 152.53, 187.53; FT-IR $v_{\max }$ : 1495, 1484, 1390, 1212 , 1162, 1083, 700, $693 \mathrm{~cm}^{-1}$; MS : m/z : 277(M+1), 150, 134(100), 109, 77.

Typical reaction of tertiary amine with (2). 2,4,6-Tribromophenyl chlorothionoformate ${ }^{13}$ (2) $(0.809 \mathrm{~g}, 1.97 \mathrm{mmol})$ was added to a solution of tertiary amine $(0.2 \mathrm{~g}, 0.273 \mathrm{ml}, 1.97 \mathrm{mmol})$ in dichloromethane $(20 \mathrm{ml})$ and the solution stirred under nitrogen at $20^{\circ} \mathrm{C}$ for 2 hours. The solvent was evaporated affording a crude oily product which was purified by chromatography (pet.ether:ethyl acetate 20:1) on silica.

$\boldsymbol{O}$-2,4,6-Tribromophenyl diethylthiocarbamate (5b). White crystals, $91 \%$ yield, m.p. $76-78^{\circ} \mathrm{C}$. ${ }^{1} \mathrm{H}$ NMR $\delta$ 1.34(t, $\left.J=7.2,3 \mathrm{H}, \mathrm{CH}_{3}\right), 1.41\left(\mathrm{t}, J=7.2,3 \mathrm{H}, \mathrm{CH}_{3}\right), 3.75\left(\mathrm{q}, J=7.2,2 \mathrm{H}, \mathrm{CH}_{2}\right)$, 3.89(q, $\left.J=7.2,2 \mathrm{H}, \mathrm{CH}_{2}\right), 7.17(\mathrm{~s}, 2 \mathrm{H}, \mathrm{Ar}) ;{ }^{13} \mathrm{C} \mathrm{NMR} \delta 11.63,13.57,44.72,48.81,119.46$, 119.84, 134.66, 148.05, 182.76; FT-IR $v_{\max }$ : 2973, 1547, 1516, 1433, 1361, 1214, 1158, 1089 , $859,709 \mathrm{~cm}^{-1} ; \mathrm{MS}: \mathrm{m} / \mathrm{z}: 446\left(\mathrm{M}^{+}\right), 366,266,100(100), 72$. 
$\boldsymbol{O}$-2,4,6-Tribromophenyl dimethylthiocarbamate (6b). White crystals, 96\% yield, m.p. 125$127^{\circ} \mathrm{C} .{ }^{1} \mathrm{H}$ NMR $\delta 3.42\left(\mathrm{~s}, 3 \mathrm{H}, \mathrm{CH}_{3}\right), 3.49\left(\mathrm{~s}, 3 \mathrm{H}, \mathrm{CH}_{3}\right), 7.72(\mathrm{~s}, 2 \mathrm{H}, \mathrm{Ar}) ;{ }^{13} \mathrm{C} \mathrm{NMR} \delta 39.14,43.71$, 119.59, 119.68, 134.72, 148.10, 183.75; FT-IR $v_{\max }: 3069,1541,1441,1389,1280,1229,1107$, $855,745,728 \mathrm{~cm}^{-1} ; \mathrm{MS}: \mathrm{m} / \mathrm{z}: 417\left(\mathrm{M}^{+}\right), 266,187,88,72(100)$.

$\boldsymbol{O}$-2,4,6-Tribromophenyl piperidine-1-carbothioate (8b). white crystals, 93\% yield, m.p. 128$130^{\circ} \mathrm{C}$ (decomposed). ${ }^{1} \mathrm{H}$ NMR $\delta 1.76\left(\mathrm{bs}, 6 \mathrm{H}, 3 \mathrm{CH}_{2}\right), 3.97\left(\mathrm{bs}, 2 \mathrm{H}, \mathrm{CH}_{2}\right), 4.10\left(\mathrm{bs}, 2 \mathrm{H}, \mathrm{CH}_{2}\right)$, 7.71(s, 2H, Ar); ${ }^{13} \mathrm{C}$ NMR $\delta 24.17,25.37,26.19,48.10,52.40,119.43,119.81,134.67,148.01$, 182.03; FT-IR $v_{\max }: 2937,1515,1439,1290,1226,1129,1087,852,741,713 \mathrm{~cm}^{-1} ; \mathrm{MS}: \mathrm{m} / \mathrm{z}$ : 458( $\left(\mathrm{M}^{+}\right), 378,266,187,112(100), 69,41$.

$\boldsymbol{O}$-2,4,6-Tribromophenyl morpholine-4-carbothioate (9b). white crystals, 83\% yield, m.p. $176-179^{\circ} \mathrm{C}$ (decomposed). ${ }^{1} \mathrm{H}$ NMR $\delta 3.81\left(\mathrm{t}, J=4.8,2 \mathrm{H}, \mathrm{CH}_{2}-\mathrm{N}\right), 3.86\left(\mathrm{t}, J=4.8,2 \mathrm{H}, \mathrm{CH}_{2}-\mathrm{N}\right)$, $4.05\left(\mathrm{t}, J=4.8,2 \mathrm{H}, \mathrm{CH}_{2}-\mathrm{O}\right), 4.17\left(\mathrm{t}, J=4.8,2 \mathrm{H}, \mathrm{CH}_{2}-\mathrm{O}\right), 7.72(\mathrm{~s}, 2 \mathrm{H}, \mathrm{Ar}) ;{ }^{13} \mathrm{C} \mathrm{NMR} \delta 47.44$, $50.75,66.30,66.38,119.60,119.77,134.77,147.79,182.94$; FT-IR $v_{\max }$ : 2859, 1515, 1434, 1282, 1246, 1220, 1194, 1113, 1032, 848, $739 \mathrm{~cm}^{-1}$; MS : m/z : 459(M $\left.\mathrm{M}^{+}\right), 380,347,266,187$, 114(100), 70, 42.

O-2,4,6-Tribromophenyl 4-chlorobutyl(methyl)carbamothioate (10b). colourless oil , 89\% yield, ${ }^{1} \mathrm{H}$ NMR $\delta$ (rotamer 1) $1.89\left(\mathrm{bs}, 4 \mathrm{H}, 2 \mathrm{CH}_{2}\right), 3.34\left(\mathrm{~s}, 3 \mathrm{H}, \mathrm{CH}_{3}\right), 3.58\left(\mathrm{t}, J=5.7,2 \mathrm{H}, \mathrm{CH}_{2}-\mathrm{N}\right)$, $3.77\left(\mathrm{t}, J=7.2,2 \mathrm{H}, \mathrm{CH}_{2}-\mathrm{Cl}\right), 7.69(\mathrm{~s}, 2 \mathrm{H}, \mathrm{Ar}) ;{ }^{1} \mathrm{H} \mathrm{NMR} \delta$ (rotamer 2$) 1.89\left(\mathrm{bs}, 4 \mathrm{H}, 2 \mathrm{CH}_{2}\right), 3.60(\mathrm{t}$, $\left.J=6.3,2 \mathrm{H}, \mathrm{CH}_{2}-\mathrm{N}\right), 3.92\left(\mathrm{t}, J=6.6,2 \mathrm{H}, \mathrm{CH}_{2}-\mathrm{Cl}\right), 7.69(\mathrm{~s}, 2 \mathrm{H}, \mathrm{Ar}) ;{ }^{13} \mathrm{C} \mathrm{NMR} \delta$ (rotamer 1$)$ 23.82, 29.44, 37.12, 44.60, 51.31, 119.60, 119.75, 134.65, 147.96, 183.42; ${ }^{13} \mathrm{C} \mathrm{NMR} \delta$ (rotamer 2) $25.31,29.73,42.13,44.75,54.65,119.62,119.82,134.70,148.07,183.69$; FT-IR $v_{\max }$ : 2941, 1524, 1440, 1403, 1286, 1226, 1090, 857, $743 \mathrm{~cm}^{-1}$; MS : m/z : 444(M $\left.\mathrm{M}^{+}\right), 364,345,266,187$, 164, 91(100).

Reaction of $\boldsymbol{N}, \boldsymbol{N}$-dimethylaniline with 2. The mixture of 2,4,6-tribromophenyl chlorothionoformate (2) $(1.68 \mathrm{~g}, 4.12 \mathrm{mmol})$ and $N, N$-dimethylaniline $(0.5 \mathrm{~g}, 0.52 \mathrm{ml}, 4.12 \mathrm{mmol})$ were heated under nitrogen at $130^{\circ} \mathrm{C}$ for 24 hours to give a blue oil. The oily product was purified by column chromatography (pet. ether:ethyl acetate 20:1) and O-2,4,6-tribromophenyl methyl(phenyl)thiocarbamate (7b) was separated as a yellow crystals (1.4g, $71 \%$ ), m.p. 127$129^{\circ} \mathrm{C} .{ }^{1} \mathrm{H}$ NMR $\delta 3.77\left(\mathrm{~s}, 3 \mathrm{H}, \mathrm{CH}_{3}\right), 7.36-7.51(\mathrm{~m}, 5 \mathrm{H}, \mathrm{Ar}-\mathrm{N}), 7.66(\mathrm{~s}, 2 \mathrm{H}, \mathrm{Ar}) ;{ }^{13} \mathrm{C} \mathrm{NMR} \delta 45.19$, 119.52, 119.64, 125.90, 128.16, 129.53, 134.65, 143.07, 148.03, 183.61; FT-IR $v_{\max }: 2922,1492$, $1437,1386,1231,1159,1085,718,693 \mathrm{~cm}^{-1}$; MS : m/z : 480(M $), 400,345,266,187,134(100)$, 106, 77.

Hydrolysis of 5a. The thiocarbamate (5a) $(0.51 \mathrm{~g}, 2 \mathrm{mmol})$ and dimethylsulfate $\quad(0.261 \mathrm{~g}$, $2.07 \mathrm{mmol})$ were refluxed under nitrogene for 3 hours in 1,2-dichloroethane $(20 \mathrm{ml})$. The solvent was evaporated affording a green oil which was washed twice with ether $(10 \mathrm{ml})$ to give the iminium salt. The salt ( $0.54 \mathrm{~g}, 2 \mathrm{mmol}$ ) was disolved in water and refluxed for 2 hours. Excess water was evaporated under reduced pressure to afford 4-chloro- $\mathrm{N}$ - methylbutan-1-amine hydrogensulfate as a spectroscopycally pure oil ( $0.33 \mathrm{~g}$ ) in $96 \%$ yield. 


\section{Acknowledgements}

The authors are grateful to the University of Urmia for support of this work.

\section{References}

1. Bradarani, M. M.; Prager, R. H. Tetrahedron Lett., 1999, 40, 7403.

2. Bradarani, M. M. ; Millan, D. S. ; Prager, R. H. J. Sci. I. R. Iran, 2001, 12, 27.; Chem. Abstr. 2002, 136, 262753.

3. Hageman, H. A. Organic Reaction. 1953, 7, 198.

4. (a) Gardmer, J.; Knoch, F. Arch. Pharm., 1921, 259, 135. (b) Flynn, E. H., Murphy, H. W.; McMahon, R. E. J. Am. Chem. Soc., 1955, 77, 3104. (c) Campbell, J. A. J. Org. Chem. 1957, 22, 1259.

5. Hobson, J. D., McCluskey, J. G. J. Chem. Soc. (C), 1967, 2015.

6. Olofson, R. A.; Martz, J. T.; Senet, J. P.; Piteau, M.; Malfroot, T. J. Org. Chem. 1984, 49, 2081.

7. Montzka, T. A.; Matiskella, J. D.; Partyka, R. A. Tetrahedron Lett. 1974, 14, 1325.

8. Olofson, R. A.; Schnur, R. C.; Bunes, L.; Pepe, J. P. Tetrahedron Lett. 1977, 17, 1567.

9. Cooley, J. H.; Evain, E. J. Synthesis, 1989, 1.

10. Von Braun, J.; Kohler, Z. Ber. 1918, 51, 255.

11. Perrin, D. D.; Armarego, W. L. F.; Perrin, D. R., Purification of Laboratory Org Chemicals, $1^{\text {st }}$ Edn., Permagan Press: Oxford, 1966.

12. Barton, D.H.R.; Jang, D.O.; Jaszberenyi, J.Cs. Tetrahedron, 1993, 49, 7194

13. Barton. D. H. R. Blundell. P. Dorchak. J. Jang. D. O. Jaszberenyi. J. Cs. Tetrahedron. 1991, 47, 8969. 